\title{
Dirty appearing white matter in the brain is associated with altered cerebrospinal fluid pulsatility and hypertension in individuals without neurologic disease
}

Clive B. Beggs PhD ${ }^{1,2}$, Christopher Magnano $\mathrm{MS}^{2,3}$, Simon J. Shepherd PhD ${ }^{1}$, Pavel Belov ${ }^{2}$, Deepa P. Ramasamy MD ${ }^{2,3}$, Jesper Hagemeier PhD ${ }^{2}$, Robert Zivadinov MD, $\mathrm{PhD}^{2,3}$

${ }^{1}$ Centre for Infection Control and Biophysics, University of Bradford, Richmond Road, Bradford BD7 1DP, UK; ${ }^{2}$ Buffalo Neuroimaging Analysis Center, Department of Neurology, School of Medicine and Biomedical Sciences, University at Buffalo, 100 High St., Buffalo, NY 14203, NY, USA $;^{3}$ MRI Clinical Translational Research Center, School of Medicine and Biomedical Sciences, University at Buffalo, Buffalo, NY, USA

Corresponding Author:

Prof Clive Beggs

Centre for Infection Control and Biophysics

School of Engineering

University of Bradford

Richmond Road

Bradford

West Yorkshire

BD7 1DP

United Kingdom

email: c.b.beggs@bradford.ac.uk, Tel: +44(0)1274 233679, Fax: +44(0)1274 234124

Short title: Dirty appearing white matter and CSF pulsatility

Type of manuscript: original research

Keywords: Dirty appearing white matter; cerebrospinal fluid; hypertension; leukoaraiosis; magnetic resonance imaging

Sources of funding: This work has been supported in part by a grant from the Annette Funicello Research Fund for Neurological Diseases.

\section{Disclosures}

Clive Beggs, Christopher Magnano, Simon Shepherd, Pavel Belov, Deepa Ramasamy and Jesper Hagemeier have nothing to disclose. Robert Zivadinov received personal compensation from Teva Pharmaceuticals, Biogen Idec, EMD Serono and Genzyme for speaking and consultant fees. Dr. Zivadinov also received financial support for research activities from Biogen Idec, Teva Pharmaceuticals, Genzyme and Novartis.

Abstract count: 247, Word count (text and references only): 4945, Number of Tables: 4, Number of Figures: 2, Number of references: 48. 


\section{Dirty appearing white matter in the brain is associated with altered cerebrospinal fluid pulsatility and hypertension in individuals without neurologic disease}

\section{Abstract}

\section{Background and purpose}

Aging of the healthy brain is characterized by focal or non-focal white matter (WM) signal abnormality (SA) changes, which are typically detected as leukoaraiosis (LA). Hypertension is a risk factor for WM lesion formation. This study investigated whether LA might be associated with increased CSF pulsatility linked to arterial hypertension.

\section{Methods}

101 individuals without neurologic diseases (53 females and 48 males) aged between 18-75 years underwent $3 \mathrm{~T}$ brain $\mathrm{MRI}$ with cine phase contrast imaging for CSF flow estimation, after providing their informed consent. LA was defined as the presence of focal T2 WM SA changes and/or non-focal uniform areas of signal increase termed dirty-appearing-whitematter (DAWM). Relevant information relating to cardiovascular risk factors was also collected.

\section{Results}

When controlled for age and hypertension, significant partial correlations were observed between: DAWM volume and: net negative flow ( $r=-0.294, p=0.014)$; net positive flow (NPF) $(r=0.406, p=0.001)$; and peak positive velocity $(r=0.342, p=0.004)$. Multiple linear regression analysis revealed DAWM volume to be significantly correlated with CSF NPF $(p=0.019)$ and hypertension ( $p=0.007$ ), whereas T2 WM SA volume was only significantly correlated with age $(p=0.002)$. Combined DAWM and T2 WM SA volumes were significantly related with age $(p=0.001)$ and CSF peak negative velocity $(p=0.041)$.

\section{Conclusions}

Rarefaction of WM leading to LA is a multifactorial process, in which formation of DAWM induced by hypertension and increased aqueductal CSF pulsatility, may play a contributory role. These two factors appear to act independently of each other in a process that is independent of age. 


\section{Introduction}

Aging of the brain in healthy individuals is characterized by atrophy and focal or non-focal white matter (WM) signal abnormality (SA) changes, which are typically detected as leukoaraiosis (LA). ${ }^{1}$ However, much remains unknown about the pathophysiology underlying these changes. WM SA changes of presumed vascular origin are a common finding in brain MRI of older individuals and contribute to cognitive and functional decline. ${ }^{2}$ Although WM degeneration is characterized pathologically by demyelination, axonal loss, and rarefaction (processes often attributed to ischemia), ${ }^{3}$ it is not known precisely how WM SA changes form. ${ }^{4}$ A recent study, investigating the intensity patterns and morphological features of periventricular WM SA changes, found hyperintensity intensity levels, distribution, and association with risk factors and disease, to be indicative of true tissue abnormalities in old age, and something that should not be dismissed as artifacts. ${ }^{4}$ In particular, the role of dirty appearing white matter (DAWM) (sometimes termed 'diffusely abnormal white matter') formation in the periventricular region is poorly understood. ${ }^{5}$ DAWM (Figure 1 ) is defined as a non-focal, uniform region of intermediate signal intensity between that of focal T2 WM SA changes and that of normal-appearing WM. Image-pathology correlation studies reveal that non-focal DAWM changes, compared with focal T2 WM SA changes, are associated with mild blood-brain barrier breakdown and myelin loss. ${ }^{6}$ Therefore, it is thought that non-focal DAWM may represent an early stage of WM pathology, ${ }^{6}$ which is followed at a later stage by focal T2 WM SA formation. ${ }^{7,8}$

Recently we undertook a study involving multiple sclerosis (MS) patients, ${ }^{9}$ in which we found WM lesion formation to be associated with increased cerebrospinal fluid (CSF) pulsatility in the aqueduct of Sylvius (AoS). Follow-up work involving healthy subjects linked increased aqueductal pulsatility with biomechanical changes in the intracranial space associated with altered cerebral venous hemodynamics. ${ }^{10}$ In addition, we found heart disease, overweight and smoking to be associated with increased prevalence of extracranial venous abnormalities, ${ }^{11}$ suggesting that cardiovascular risk factors might also influence pulsatility within the intracranial space.

It has been postulated that the presence of cardiovascular comorbidities, in particular arterial hypertension (a known risk factor for WM SA formation) ${ }^{12}$ might be linked to increased CSF pulsatility. ${ }^{13}$ Hypertension is associated with reduced vascular compliance, ${ }^{14}$ particularly in smaller arterial vessels. ${ }^{15}$ This tends to promote increased pulsatility in the cerebral vascular bed, ${ }^{16,17}$ something that is thought to cause endothelial damage and lumenal change. ${ }^{14}$ Increased cerebral blood flow pulsatility has been found to be associated with microstructural changes in the WM. ${ }^{16,18,19}$ Therefore, given that increased CSF pulsatility in the AoS is thought to be associated with greater pulsation in the cerebral vascular bed, ${ }^{20}$ it can be 
postulated that increased aqueductal CSF pulsatility might also be associated with DAWM formation.

We therefore hypothesized that DAWM formation and WM SA changes in the brain might be associated with increased aqueductal CSF pulsatility linked to arterial hypertension. In order to test this hypothesis, we undertook a study involving 101 individuals without neurologic diseases with the aim of establishing whether or not WM SA changes, and in particular the presence of DAWM, are associated with increased aqueductal pulsatility linked to cardiovascular comorbidities.

\section{Methods}

\section{Patient population}

This study utilized data from an ongoing prospective study of genetic and environmental risk factors in individuals with and without neurologic diseases that enrolled over 1,100 subjects. ${ }^{21,22}$ One hundred and one consecutive individuals without neurologic diseases (53 females and 48 males) aged between 18 to 75 years who underwent magnetic resonance imaging (MRI) scan with cine phase contrast imaging for CSF flow estimation were included. The individuals needed to qualify on a health screening questionnaire containing information about medical history (illnesses, surgeries, medications, etc.) and meet the health screen requirements for $\mathrm{MRI}$ on physical examination, as previously described. ${ }^{22,23,24}$ Exclusion criteria were: pre-existing medical conditions known to be associated with brain pathology (e.g. cerebrovascular disease, positive history of alcohol abuse, etc.), history of cerebral congenital vascular malformations, or pregnancy. Relevant information relating to cardiovascular risk factors [body mass index (BMI), hypertension, hypercholesterolemia, heart disease, diabetes and smoking] was collected.

All participants underwent clinical and MRI examinations. The study was approved by the University of Buffalo Institutional Review Board and written informed consent was obtained from all subjects.

\section{MRI acquisition and analysis}

The subject's brains were examined on a 3 Tesla GE Signa Excite HD 12.0 Twin Speed scanner (General Electric, Milwaukee, WI). All sequences were run on an 8-channel head and neck (HDNV) coil. All analyses were performed in a blinded manner.

Brain MRI sequences included 3D T1-weighted imaging (WI) using a fast spoiled gradient echo (FSPGR) with magnetization-prepared inversion recovery (IR) pulse for segmentation; 
cine phase contrast imaging for CSF flow estimation; multi-planar dual fast spin-echo proton density (PD) and T2-WI; and fluid attenuated inversion recovery (FLAIR) for lesion analysis. A sagittal T2-weighted fast SE sequence was also acquired as a localizer for the cine phase contrast (PC) prescription, as previously described, with the cine PC sequence prescribed as an oblique axial slice through the AoS. ${ }^{9}$

Pulse sequence characteristics for 3T MRI were as follows: all scans were acquired with a $256 \times 256$ matrix and a $25.6 \mathrm{~cm}$ FOV for an in-plane resolution of $1 \times 1 \mathrm{~mm}^{2}$ with a phase FOV of $75 \%$ and one average. Sequence-specific parameters were as follows: for 3D HIRES, T1-WI were Echo Time/Inversion Time/Repetition Time TE/TI/TR=2.8/900/5.9 ms, Flip Angle $(F A)=10^{\circ}$, and $1 \mathrm{~mm}^{3}$ isotropic voxels; for the PD/T2: 3-mm-thick slices with no gap, TE1/TE2/TR $=12 / 95 / 3000 \mathrm{~ms}, \mathrm{ETL}=14$, and for the FLAIR scans, 3-mm-thick slices with no gap, TE/TI/TR=120/2100/8500 ms.

CSF flow quantification was performed using a single slice cine phase-contrast velocityencoded pulse-gated gradient echo sequence (cine PC) with an TE/TR of 7.9/40 ms, a slice thickness of $4 \mathrm{~mm}$, a velocity encoding of $20 \mathrm{~cm} / \mathrm{s}$ and 32 phases acquired corresponding to the cardiac cycle. All subjects underwent the MRI exam during the same time of day (in the afternoon hours) to control for circadian variation. The cine PC sequence was acquired with a resolution of $0.39 \times 0.39 \times 4 \mathrm{~mm}^{3}$, with the AoS in the center of the FOV, such that the wrap around artifact was present in the edges of the FOV, but did not overlap with the desired region of interest $(\mathrm{ROI})$.

\section{Cine phase contrast image analysis}

The net positive and net negative flows (NPF and NNF), together with the net flow (NF = $\mathrm{NNF}+\mathrm{NPF}$ ) and the peak positive and negative velocities (PPV and PNV) were calculated using a validated methodology as previously described. ${ }^{9}$ The CSF flow data was processed using GE ReportCard software (version 3.6; General Electric, GE, Milwaukee, WI) and positive and negative velocities over all 32 phases were recorded. In order to overcome limitations in the ReportCard software an in-house semi-automated minimum area of contour change (MACC) program was used to correct the ROls for each phase, as previously described. ${ }^{9}$ NPF and NNF were calculated using only the phases, which have positive and negative velocities, respectively. ${ }^{9} \mathrm{CSF}$ flow measures are presented in microliters per beat $\left(\mu \mathrm{L} /\right.$ beat, $\left.1 \mu \mathrm{L}=1 \mathrm{~mm}^{3}\right)$, while CSF velocity measures are presented in $\mathrm{cm} / \mathrm{s}$. CSF flow direction was calculated based on slice prescription such that flow through the AoS out of the slice (during diastole, towards the third ventricle) was given as positive, whereas flow into the slice (during systole, towards the fourth ventricle) was negative, as described previously. ${ }^{9}$ 


\section{Detection of white matter signal abnormality changes:}

LA was defined as the presence of focal T2 WM SA changes and/or non-focal uniform areas of signal increase termed DAWM. Each subject's relevant T2/PD images were co-registered to the FLAIR using a rigid body (6-degrees-of-freedom) registration. ${ }^{25}$ The FLAIR image was used to outline T2 WM SAs, while the co-registered T2/PD images were used to confirm the presence of T2 WM SAs and to check for infratentorial T2 WM SAs, as FLAIR imaging is known to be insensitive in this area. The T2 WM SA number and volume (T2 WM-SAV) were outlined using a semi-automated edge detection contouring/thresholding technique as described previously. ${ }^{26}$ All T2 WM SAs were divided into individual ROls $\geq 3 \mathrm{~mm}$ in size (equivalent to $\geq 14.1 \mathrm{~mm} 3$ ). The periventricular and deep WM regional localization of T2 WM SAs was also determined.

In addition, areas of DAWM were outlined (Figure 1). The DAWM was defined as a uniform, non-focal area of signal increase on the FLAIR/T2/PD-weighted sequence at 3T, with a subtly increased signal intensity compared with the contralateral signal intensity of normal appearing WM but less than that of T2 WM SAs, as previously proposed. ${ }^{27}$

\section{Statistical analysis}

Analysis was undertaken using in-house algorithms written in $R$ (open source statistical software) and Matlab (Mathworks, Natick, Mass). A one-way ANOVA was used to analyze changes in CSF pulsatility with age, with values of $p<0.05$ using a two-tailed test considered statistically significant.

Correlation (full and partial) matrices (Spearman's $r$ ) were computed to quantify the relationships between the variables. Multiple linear regression analysis using the dependent variables T2 WM-SAV, DAWM volume, DAWM volume+T2 WM-SAV, T2 periventricular SA number (T2 PVL-SAN) and T2 deep WM SA number (T2 DWM-SAN) was also performed. In this analysis the independent variables were age, BMI, hypertension, hypercholesterolemia, heart disease, diabetes, smoking, NF, NNF, NPF, PPV and PNV. A stepwise approach was taken, with variables excluded according to their respective $t$ values. Only significant variables with $p<0.05$ were included in the final regression models.

Values of $p<0.05$ using a two-tailed test were considered statistically significant after the Benjamini-Hochberg ${ }^{28}$ correction for multiple comparisons was applied. 


\section{Results}

\section{Demographic and clinical characteristics}

Table 1 shows the demographic, clinical and MRI characteristics. Subject average age was 44.7 years (range: 18 - 75 years), with females comprising $54.5 \%$ of the cohort. Recorded cardiovascular risk factors were as follows: $8.2 \%$ had a history of cardiovascular disease; $22.8 \%$ were smokers; $27.8 \%$ had hypertension; $17.0 \%$ had hypercholesterolemia; with $6.2 \%$ having diabetes. The mean BMI was $26.8 \mathrm{~kg} / \mathrm{m}^{2}$. With the exception of smoking, no significant differences were observed between the male and female subjects. The MRI characteristics are also presented in Table 1.

One-way ANOVA of the CSF data grouped according to age (Figure 2), revealed statistical differences for NF ( $p=0.003)$, NPF $(p=0.021)$ and NPV $(p=0.030)$, suggesting that the values of these variables changed with age.

\section{Correlation analysis}

Correlation analysis of the demographic and cardiovascular risk factor variables against the MRI variables (Table 2) revealed age to be significantly correlated with NF, PNV, T2 WMSAV, DAWM volume and DAWM volume+T2 WM-SAV, while hypertension was significantly correlated with T2 WM-SAV, DAWM volume and DAWM volume+T2 WM-SAV, with BMI significantly correlated with T2 WM-SAV.

Correlation analysis of the relationships between the MRI variables (Table 3a) revealed significant correlations between the CSF variables NNF, NPF, PPV and PNV, and the MRI variables T2 WM-SAV, DAWM volume and DAWM volume+T2 WM-SAV. Significant observations were also observed between: T2 DWM-SAN and the variables NNF, NPF and PPV; and T2 PVL-SAN and NPF.

\section{Partial correlation analysis}

When controlled for age and hypertension, the partial correlation analysis (Table $3 b$ ) revealed significant correlations between: DAWM volume and NNF, NPF and PPV; DAWM volume+T2 WM-SAV and NPF and PPV; and T2 DWM-SAN and NNF, NPF.

\section{Linear regression analysis}

Multiple linear regression analysis was undertaken with T2-SAV, DAWM volume, DAWM volume+T2 WM-SAV, T2 PVL-SAN and T2 DWM-SAN as dependent variables. The results of this analysis (Table 4) revealed that significant models were only achieved for T2 WMSAV, DAWM volume, and the composite variable DAWM volume+T2 WM-SAV. Interestingly, there were marked differences between the models for T2 WM-SAV and DAWM volume. For 
the dependent variable T2 WM-SAV, the only significant correlation was age, whereas for DAWM volume, hypertension and NPF were significant. The results for the DAWM volume+T2 WM-SAV model reflected the composite nature of that variable, with age and PNV both being significant.

\section{Discussion}

Our principal finding is that in individuals without neurologic diseases, DAWM formation appears to be associated with both hypertension and increased aqueductal CSF pulsatility, rather than aging per se. When controlled for age, the partial correlations between DAWM volume and the CSF variables, NNF, NPF, PPV and PNV were all significant, with the partial correlation between DAWM volume and hypertension approaching significance. This suggests that rarefaction of the WM leading to LA is a multifactorial process, in which formation of DAWM is influenced by factors other than age. As such, our findings mirror those of Jolly et al. ${ }^{19}$ who found microstructural WM changes to be independent of age and associated with increased cerebral blood flow pulsatility and aqueductal CSF pulse volume.

Cerebral WM SA changes frequently occur in healthy ageing and are typically detected as $L A$, a radiological finding that presents as increased signal intensity on T2-weighted MR images. WM SA changes are a common finding in brain MRI of older individuals and are thought to contribute to cognitive and functional decline. ${ }^{2}$ While it is not known precisely how these WM SA changes form, it has been shown that periventricular WM hyperintensities in old age represent true tissue abnormalities. ${ }^{4}$ WM degeneration is characterized pathologically by demyelination, axonal loss, and rarefaction, which are thought to be related to ischemia. ${ }^{3}$ Histological studies have shown LA to be characterized by WM morphological changes around the periventricular veins. ${ }^{29,30}$ In particular, LA is associated with noninflammatory collagenosis of the periventricular veins, resulting in thickening of the vessel walls and narrowing of the lumen. ${ }^{29}$ Vascular dementia, closely related to LA, has been shown to be associated with alterations in venous pulsation, ${ }^{31}$ and it has been postulated that the thickening of the vein walls in LA may be a protective response against increased vascular pulsatility. ${ }^{29}$ Other subtle changes appear to be associated with increased vascular pulsatility. Jolly et al. ${ }^{19}$ found fractional diffusion anisotropy to be negatively correlated with both arterial pulse and pulsatility in deep venous territories. Changes in diffusion anisotropy have also been reported in the normal-appearing white matter around LA lesions ${ }^{32}$ and it is thought that these represent early changes in the structural organization of white matter that are likely to precede the emergence of LA. ${ }^{19}$ 
DAWM is poorly understood and appears something of an enigma. Most of the work undertaken on DAWM has been done in the context of MS, where it has been shown that quantitative MR imaging measures of DAWM lie between those of normal-appearing WM and focal WM lesions. ${ }^{33,34}$ This has led some to suggest that DAWM may represent an early stage of WM pathology, ${ }^{6}$ which is followed at a later stage by focal WM lesion formation. In support of this, Chung et al. ${ }^{35}$ reported that DAWM appears to be converted to T2 WM lesions in Alzheimer's disease (AD) patients who experience severe jugular venous reflux. Others however, suggest that DAWM is by no means a preceding stage for focal lesion formation, but rather a different pathologic phenomenon altogether, arising from secondary Wallerian degradation and resulting in extensive axonal and myelin loss. ${ }^{34,36}$

While the pathology associated with DAWM remains unclear, there appears to be a consensus that DAWM reflects an increase in extracellular water within the brain parenchymal tissue. ${ }^{33}$ The apparent diffusion coefficient (ADC) has been shown to be greater in DAWM compared with normal-appearing WM in patients with $M S,{ }^{34}$ indicating the presence of microstructural changes in DAWM that permit increased transport of water. Laule et al. ${ }^{37}$ found that the primary abnormality in DAWM was a reduction or perturbation of the myelin phospholipids that was associated with a reduction in the myelin water fraction. This was accompanied by an increase in the total water content of the DAWM, probably due to an influx of water into spaces formerly occupied by the phospholipids and myelin proteins. As such, this suggests that profound microstructural changes may occur within DAWM, possibly due to lipid abnormalities. ${ }^{38}$ While DAWM has been studied in MS, it has rarely been investigated outside of this context, and so it cannot be assumed that DAWM formation in healthy individuals necessarily represents the same pathophysiology as that observed in MS patients. Notwithstanding this, given that water has a long T2 value, it is reasonable to postulate that DAWM formation might also be associated with increased ADC in healthy subjects.

While the specific impact of altered CSF pulsatility on DAWM formation has not previously been investigated, a number of studies have linked increased intracranial fluid pulsatility and microstructural WM damage. ${ }^{16,18,19}$ Our finding that DAWM formation is associated with hypertension appears to be consistent with those who linked microstructural WM damage with increased cerebral blood flow pulsatility. ${ }^{16,18,19}$ Increased pulsatility is indicative of decreased arterial compliance, something that is associated with arteriosclerosis ${ }^{39}$ and hypertension. ${ }^{14}$ Hypertension is a risk factor for small vessel disease ${ }^{40}$ and $L A,{ }^{41}$ and is associated with changes in vascular mechanics. ${ }^{14,16}$ It has been suggested that greater vascular pulsatility might cause WM damage by increasing perivascular shear stress and 
inducing demyelination, resulting in microstructural WM changes and contributing to the proliferation of LA over time. ${ }^{19}$ Stiffening of the aorta has been linked to the transmission of excessive flow pulsatility into the brain, ${ }^{16,17}$ something that is associated with WM microstructural changes in healthy older individuals. ${ }^{19}$ Recently, Tarumi et al. ${ }^{17}$ demonstrated that arterial stiffness in aging is positively correlated with cerebral vascular pulsatility, and that this in turn is associated with a greater volume of WM hyperintensities. Excessive intracranial cardiac-related pulsatility (cerebral arterial and CSF) has also been associated with brain atrophy among elderly individuals. ${ }^{18}$ Microstructural changes associated with increased cerebral pulsatility may therefore represent early stage alterations in the structural organization of the WM, likely to precede the emergence of LA. ${ }^{19}$ In our linear regression model the predictors NPF and hypertension were only able to account for about $20 \%$ of the variance associated with DAWM formation. This suggests that although both increased aqueductal pulsatility and hypertension are associated with DAWM formation, other unknown contributory factors must be at work.

The intracranial CSF windkessel mechanism dampens the vascular pulse so that blood flow through the cerebral capillary bed is non-pulsatile. ${ }^{42}$ If however this mechanism becomes dysfunctional, then this might lead to increased pulsatility in the AoS, as postulated by Greitz, ${ }^{20}$ who argued that pulsations in the cerebral capillaries were transmitted through the parenchyma to the lateral ventricles. If this were the case, then one might expect aqueductal pulsatility to be correlated with hypertension. But when we controlled for age, we did not find any significant correlations between hypertension and the CSF variables, suggesting that the two phenomena are not linked. As such, our finding supports Rashid et al. ${ }^{43}$ who found in a rat study that elevated pulsatile CSF flow in the aqueduct was not matched by an increase in microvascular pulsatile flow. Furthermore, our regression analysis model indicated that both hypertension and NPF were significant predictors of DAWM formation, suggesting that the two mechanisms may be acting independently of each other to produce WM SA changes. Increased aqueductal pulsatility has been linked with constricted cerebral venous outflow in healthy individuals ${ }^{10}$ and MS patients, ${ }^{44}$ suggesting that this phenomenon may be more associated with altered compliance in the sub-arachnoid space, rather than pulsatility in the cerebral vascular bed. Notwithstanding this, a number of researchers have observed a link between the aqueductal pulsatility and subtle changes in the brain parenchyma. Daouk et al. ${ }^{45}$ found ADC, an early indicator of microstructural changes, to be strongly correlated with aqueductal stroke volume in $A D$, and Jolly et al. ${ }^{19}$ found increased aqueductal CSF pulse volume to be associated with microstructural WM changes in elderly subjects. Having said this, these correlations appear to be confined microstructural changes, with Jolly et al finding no association between CSF pulse volume and LA severity. ${ }^{19}$ 
The results regarding T2 WM SA formation appear more ambiguous than those for DAWM formation. The regression analysis model for T2 WM-SAV identified age as the only significant predictor, while those for T2 PVL-SAN and T2 DWM-SAN revealed no significant predictors. However, care should be taken before completely ruling out other contributory factors to T2 WM SA formation. This is because the NPF and NF characteristics of the $>70$ age group were markedly different to those $<70$ years of age (Figure 2). When we repeated the multiple regression analysis using only those subjects $<70$ years of age, we found T2 WM-SAV to be significantly correlated with hypertension rather than age (statistical power = $80.9 \%)$. Also, in the younger cohort, DAWM volume+T2 WM-SAV was significantly correlated with hypertension and NPF (statistical power $=99.2 \%$ ), and DAWM volume was significantly correlated with hypertension and NPF (statistical power $=99.7 \%$ ). This suggests that hypertension may be influential in both T2 WM SA and DAWM formation in young and middle-aged adults. As a risk factor for both small vessel disease ${ }^{40}$ and $L A,{ }^{41}$ it is perhaps not surprising that we found hypertension associated with T2 WM-SA and DAWM formation. LA is characterized by thickening and hardening of vessel walls. ${ }^{46}$ This in theory should cause blood flow in the cerebral vascular bed to become more pulsatile. ${ }^{16,17}$ Bateman $^{42}$ found blood flow through the WM to be highly pulsatile in individuals with LA and concluded that this would increase endothelial shear stress, and in turn cause WM damage. ${ }^{31}$ Lumenal narrowing associated with LA may also influence cerebral vascular density. LA is associated with decreased cerebral blood flow and capillary loss in both the cortex and the normally appearing $\mathrm{WM}^{46}$ This suggests that the condition affects the brain globally and that it is associated with chronic ischemia. ${ }^{46}$ While the physiological mechanisms associated with this phenomenon are not understood, recent work by Hall et al. ${ }^{47}$ has shown that ischemia associated with reduced arteriolar perfusion can result in constriction of capillaries by adjacent pericytes, rapidly leading to pericyte death and permanent occlusion of the capillaries. $^{48}$

While we found an association between aqueductal CSF pulsatility and microstructural WM changes, we did not measure the pulsatility of the cerebral blood flow. We therefore cannot assess the contribution that increased pulsatility in the cerebral vascular bed might make towards DAWM formation, or indeed, the relationship between vascular pulsatility and CSF pulsatility in the AoS.

Although we report an association between increased aqueductal pulsatility and DAWM formation in adults without neurologic diseases, previous studies have linked increased aqueductal pulsatility with: early stage WM changes ${ }^{19}$ in elderly individuals; microstructural WM changes in AD patients; ${ }^{45}$ and increased T2 and T1 WM-SAV in MS patients. ${ }^{9}$ As such there appears to be an association between WM damage and aqueductal pulsatility, which is 
independent of age. Therefore, we conclude that rarefaction of WM leading to LA is a multifactorial process, in which formation of DAWM induced by hypertension and increased aqueductal CSF pulsatility, may play a contributory role. 


\section{References}

1 Drayer BP. Imaging of the aging brain. Part I. Normal findings. Radiology 1988;166:785-796.

2 Maniega SM, Valdes Hernandez MC, Clayden JD, et al. White matter hyperintensities and normal-appearing white matter integrity in the aging brain. Neurobiol Aging 2015;36:909-918.

3 Pantoni L, Garcia JH. Pathogenesis of leukoaraiosis: a review. Stroke 1997;28:652659.

$4 \quad$ Valdes Hernandez MC, Piper RJ, Bastin ME, et al. Morphologic, distributional, volumetric, and intensity characterization of periventricular hyperintensities. AJNR Am J Neuroradiol 2014;35:55-62.

5 Ge Y, Grossman RI, Babb JS, He J, Mannon LJ. Dirty-appearing white matter in multiple sclerosis: volumetric MR imaging and magnetization transfer ratio histogram analysis. AJNR Am J Neuroradiol 2003;24:1935-1940.

6 Moore GR, Laule C, Mackay A, et al. Dirty-appearing white matter in multiple sclerosis: preliminary observations of myelin phospholipid and axonal loss. J Neurol 2008;255:1802-1811, discussion 1812.

7 Fazekas F, Chawluk JB, Alavi A, Hurtig HI, Zimmerman RA. MR signal abnormalities at $1.5 \mathrm{~T}$ in Alzheimer's dementia and normal aging. AJR Am J Roentgenol 1987;149:351-356.

8 De Groot CJ, Bergers E, Kamphorst W, et al. Post-mortem MRI-guided sampling of multiple sclerosis brain lesions: increased yield of active demyelinating and (p)reactive lesions. Brain 2001;124:1635-1645.

9 Magnano C, Schirda C, Weinstock-Guttman B, et al. Cine cerebrospinal fluid imaging in multiple sclerosis. J Magn Reson Imaging 2012;36:825-834.

10 Beggs CB, Magnano C, Shepherd SJ, et al. Aqueductal cerebrospinal fluid pulsatility in healthy individuals is affected by impaired cerebral venous outflow. J Magn Reson Imaging 2013;doi: 10.1002/jmri.24468.

11 Dolic K, Weinstock-Guttman B, Marr K, et al. Heart disease, overweight, and cigarette smoking are associated with increased prevalence of extra-cranial venous abnormalities. Neurol Res 2012;34:819-827.

12 de Leeuw FE, de Groot JC, Oudkerk M, et al. Hypertension and cerebral white matter lesions in a prospective cohort study. Brain 2002;125:765-772.

13 Krauss JK, Regel JP, Vach W, Droste DW, Borremans JJ, Mergner T. Vascular risk factors and arteriosclerotic disease in idiopathic normal-pressure hydrocephalus of the elderly. Stroke 1996;27:24-29.

14 Safar ME, Levy BI, Struijker-Boudier H. Current perspectives on arterial stiffness and pulse pressure in hypertension and cardiovascular diseases. Circulation 2003;107:2864-2869.

15 Baumbach GL, Heistad DD. Remodeling of cerebral arterioles in chronic hypertension. Hypertension 1989;13:968-972.

16 Mitchell GF, van Buchem MA, Sigurdsson S, et al. Arterial stiffness, pressure and flow pulsatility and brain structure and function: the Age, Gene/Environment Susceptibility--Reykjavik study. Brain 2011;134:3398-3407.

17 Tarumi T, Ayaz Khan M, Liu J, et al. Cerebral hemodynamics in normal aging: central artery stiffness, wave reflection, and pressure pulsatility. J Cereb Blood Flow Metab 2014;34:971-978.

18 Wahlin A, Ambarki K, Birgander R, Malm J, Eklund A. Intracranial pulsatility is associated with regional brain volume in elderly individuals. Neurobiol Aging 2014;35:365-372.

19 Jolly TA, Bateman GA, Levi CR, Parsons MW, Michie PT, Karayanidis F. Early detection of microstructural white matter changes associated with arterial pulsatility. Front Hum Neurosci 2013;7:782.

20 Greitz D. Radiological assessment of hydrocephalus: new theories and implications for therapy. Neurosurg Rev 2004;27:145-165; discussion 166-147. 
21 O'Connor K, Weinstock-Guttman B, Carl E, Kilanowski C, Zivadinov R, Ramanathan M. Patterns of dietary and herbal supplement use by multiple sclerosis patients. $J$ Neurol 2012;259:637-644.

22 Zivadinov R, Marr K, Cutter G, et al. Prevalence, sensitivity, and specificity of chronic cerebrospinal venous insufficiency in MS. Neurology 2011;77:138-144.

23 Zivadinov R, Cutter G, Marr K, et al. No association between conventional brain MR imaging and chronic cerebrospinal venous insufficiency in multiple sclerosis. AJNR Am J Neuroradiol 2012;33:1913-1917.

24 Dolic K, Weinstock-Guttman B, Marr K, et al. Risk factors for chronic cerebrospinal venous insufficiency (CCSVI) in a large cohort of volunteers. PLoS One $2011 ; 6: e 28062$.

25 Jenkinson M, Bannister P, Brady M, Smith S. Improved optimization for the robust and accurate linear registration and motion correction of brain images. Neuroimage 2002;17:825-841.

26 Zivadinov R, Heininen-Brown M, Schirda CV, et al. Abnormal subcortical deep-gray matter susceptibility-weighted imaging filtered phase measurements in patients with multiple sclerosis: a case-control study. Neuroimage 2012;59:331-339.

27 Chung CP, Beggs C, Wang PN, et al. Jugular Venous Reflux and White Matter Abnormalities in Alzheimer's Disease: A Pilot Study. J Alzheimers Dis 2013;doi: 10.3233/JAD-131112.

28 Benjamini $\mathrm{Y}$, Hochberg Y. Controlling the false discovery rate: a practical and powerful approach to multiple testing. Journal of the Royal Statistical Society $B$ 1995;57:289-300.

29 Moody DM, Brown WR, Challa VR, Anderson RL. Periventricular venous collagenosis: association with leukoaraiosis. Radiology 1995;194:469-476.

30 Brown WR, Moody DM, Thore CR, Anstrom JA, Challa VR. Microvascular changes in the white mater in dementia. J Neurol Sci 2009;283:28-31.

31 Bateman GA, Levi CR, Schofield P, Wang Y, Lovett EC. The venous manifestations of pulse wave encephalopathy: windkessel dysfunction in normal aging and senile dementia. Neuroradiology 2008;50:491-497.

32 O'Sullivan M, Summers PE, Jones DK, Jarosz JM, Williams SC, Markus HS. Normalappearing white matter in ischemic leukoaraiosis: a diffusion tensor MRI study. Neurology 2001;57:2307-2310.

33 West J, Aalto A, Tisell A, et al. Normal appearing and diffusely abnormal white matter in patients with multiple sclerosis assessed with quantitative MR. PLoS One 2014;9:e95161.

34 Vrenken H, Seewann A, Knol DL, Polman CH, Barkhof F, Geurts JJ. Diffusely abnormal white matter in progressive multiple sclerosis: in vivo quantitative MR imaging characterization and comparison between disease types. AJNR Am J Neuroradiol 2010;31:541-548.

35 Beggs $\mathrm{C}$, Chung $\mathrm{CP}$, Bergsland $\mathrm{N}$, et al. Jugular venous reflux and brain parenchyma volumes in elderly patients with mild cognitive impairment and Alzheimer's disease. BMC Neurol 2013;13:157.

36 Seewann A, Vrenken $\mathrm{H}$, van der Valk $\mathrm{P}$, et al. Diffusely abnormal white matter in chronic multiple sclerosis: imaging and histopathologic analysis. Arch Neurol 2009;66:601-609.

37 Laule $\mathrm{C}$, Vavasour IM, Leung E, et al. Pathological basis of diffusely abnormal white matter: insights from magnetic resonance imaging and histology. Mult Scler 2011;17:144-150.

38 Laule C, Pavlova V, Leung E, et al. Diffusely abnormal white matter in multiple sclerosis: further histologic studies provide evidence for a primary lipid abnormality with neurodegeneration. J Neuropathol Exp Neurol 2013;72:42-52.

39 Henry-Feugeas MC. Intracranial MR dynamics in clinically diagnosed Alzheimer's disease: the emerging concept of "pulse wave encephalopathy". Curr Alzheimer Res 2009;6:488-502.

40 Gons RA, de Laat KF, van Norden AG, et al. Hypertension and cerebral diffusion tensor imaging in small vessel disease. Stroke 2010;41:2801-2806. 
41 van Gijn J. Leukoaraiosis and vascular dementia. Neurology 1998;51:S3-8.

42 Bateman GA. Pulse-wave encephalopathy: a comparative study of the hydrodynamics of leukoaraiosis and normal-pressure hydrocephalus. Neuroradiology 2002;44:740-748.

43 Rashid S, McAllister JP, 2nd, Yu Y, Wagshul ME. Neocortical capillary flow pulsatility is not elevated in experimental communicating hydrocephalus. J Cereb Blood Flow Metab 2012;32:318-329.

44 Zamboni P, Menegatti E, Weinstock-Guttman B, et al. The severity of chronic cerebrospinal venous insufficiency in patients with multiple sclerosis is related to altered cerebrospinal fluid dynamics. Funct Neurol 2009;24:133-138.

45 Daouk J, Chaarani B, Zmudka J, et al. Relationship between cerebrospinal fluid flow, ventricles morphology, and DTI properties in internal capsules: differences between Alzheimer's disease and normal-pressure hydrocephalus. Acta Radiol 2013.

46 Brown WR, Moody DM, Thore CR, Challa VR, Anstrom JA. Vascular dementia in leukoaraiosis may be a consequence of capillary loss not only in the lesions, but in normal-appearing white matter and cortex as well. J Neurol Sci 2007;257:62-66.

47 Hall CN, Reynell C, Gesslein B, et al. Capillary pericytes regulate cerebral blood flow in health and disease. Nature 2014;508:55-60.

48 Greif DM, Eichmann A. Vascular biology: Brain vessels squeezed to death. Nature 2014;508:50-51. 


\section{Tables}

Table 1. Descriptive statistics of the demographic, cardiovascular risk factors and MRI data.

\begin{tabular}{|l|cccc}
\hline Variables & $\begin{array}{c}\text { Subjects } \\
\mathbf{n}=\mathbf{1 0 1}\end{array}$ & $\begin{array}{c}\text { Males } \\
\mathbf{n}=\mathbf{4 6}\end{array}$ & $\begin{array}{c}\text { Females } \\
\mathbf{n}=\mathbf{5 5}\end{array}$ & $\begin{array}{c}\text { Significance } \\
\mathbf{p} \text { value }\end{array}$ \\
Age (years); mean (SD) & $44.7(17.8)$ & $41.8(16.1)$ & $47.0(19.0)$ & 0.145 \\
BMI (kg/m²); mean (SD) & $26.8(5.7)$ & $27.1(5.0)$ & $26.6(6.2)$ & 0.257 \\
Current smokers; $\mathrm{n}(\%)$ & $23(22.8)$ & $15(32.6)$ & $8(14.5)$ & $0.031^{*}$ \\
Diabetes; $\mathrm{n}(\%)$ & $6(6.2)$ & $2(4.5)$ & $4(7.5)$ & $0.541^{*}$ \\
Hypertension; $\mathrm{n}(\%)$ & $20(27.8)$ & $5(17.9)$ & $15(34.1)$ & $0.134^{*}$ \\
Hypercholesterolemia; $\mathrm{n}(\%)$ & $8(17.0)$ & $2(12.5)$ & $6(19.4)$ & $0.553^{*}$ \\
Cardiovascular disease; $\mathrm{n}(\%)$ & $8(8.2)$ & $4(8.9)$ & $4(7.5)$ & $0.809^{*}$ \\
NF ( $\mu$ L/beat); mean (SD) & $-3.33(7.83)$ & $-4.35(7.84)$ & $-2.47(7.79)$ & 0.392 \\
NNF ( $\mu$ L / beat); mean (SD) & $-30.16(18.73)$ & $-31.17(22.79)$ & $-29.32(14.68)$ & 0.631 \\
NPF ( $\mu$ L / beat); mean (SD) & $26.83(18.82)$ & $26.82(20.32)$ & $26.85(17.66)$ & 0.675 \\
PPV (cm/s); mean (SD) & $6.64(3.01)$ & $6.87(3.54)$ & $6.44(2.50)$ & 0.865 \\
PNV (cm/s); mean (SD) & $-8.15(3.26)$ & $-8.32(3.67)$ & $-8.00(2.91)$ & 0.959 \\
T2 WM-SAV; mean (SD) & $350.5(1492.7)$ & $177.4(646.1)$ & $495.3(1931.6)$ & 0.319 \\
DAWM volume; mean (SD) & $903.1(1113.2)$ & $1037.0(1355.4)$ & $789.0(852.0)$ & 0.463 \\
DAWM+T2 WM-SAV; mean (SD) & $1257.1(2049.9)$ & $1214.4(1785.2)$ & $1293.5(2267.2)$ & 0.729 \\
T2 PVL-SAN; mean (SD) & $0.35(1.25)$ & $0.28(0.78)$ & $0.40(1.55)$ & 0.586 \\
T2 DWM-SAN; mean (SD) & $3.93(8.58)$ & $2.93(7.92)$ & $4.76(9.07)$ & 0.146 \\
\hline
\end{tabular}

BMI, body mass index; NF, net flow; NNF, net negative flow; NPF, net positive flow; PPV, peak positive velocity; PNV, peak negative velocity; WM-SAV, white matter signal abnormality volume; DAWM, dirty appearing white matter; PVL-SAN, periventricular signal abnormality number; DWMSAN, deep white matter signal abnormality number.

Volumes are expressed in cubic millimetres.

$p$ value determined by 2-tailed Mann Whitney U-test unless otherwise stated.

${ }^{*} p$ value determined using chi square test. 
Table 2. Spearman correlation values ( $r$ values) between demographic and cardiovascular risk factor and MRI variables $(n=101)$

\begin{tabular}{|c|c|c|c|c|c|c|c|c|c|c|}
\hline & NF & NNF & NPF & PPV & PNV & $\begin{array}{c}\text { T2 WM- } \\
\text { SAV }\end{array}$ & $\begin{array}{l}\text { DAWM } \\
\text { volume }\end{array}$ & $\begin{array}{l}\text { DAWM+T2 } \\
\text { WM-SAV }\end{array}$ & $\begin{array}{c}\text { T2 PVL- } \\
\text { SAN }\end{array}$ & $\begin{array}{c}\text { T2 DWM- } \\
\text { SAN }\end{array}$ \\
\hline Age & $0.286^{* * \star \Lambda}$ & -0.120 & $0.250^{*}$ & 0.158 & $-0.275^{\star \star \star \wedge}$ & $0.507^{* * \star \wedge}$ & $0.353^{* * \star \wedge}$ & $0.419^{* * \star \Lambda}$ & 0.108 & 0.145 \\
\hline BMI & 0.203 & -0.120 & 0.217 & 0.061 & -0.141 & $0.338^{* * \star \wedge}$ & 0.072 & 0.134 & 0.175 & 0.206 \\
\hline Smoker & -0.123 & 0.087 & -0.113 & -0.020 & 0.036 & -0.024 & 0.071 & 0.075 & 0.005 & -0.112 \\
\hline Diabetes & 0.057 & -0.147 & 0.182 & 0.122 & -0.080 & 0.069 & 0.043 & 0.044 & 0.013 & -0.029 \\
\hline Hypertension & 0.185 & -0.127 & 0.182 & $0.260^{*}$ & $-0.267^{*}$ & $0.485^{\star * \star \Lambda}$ & $0.325^{\star * \star \wedge}$ & $0.353^{* * \star \Lambda}$ & 0.115 & 0.124 \\
\hline $\begin{array}{l}\text { Hypercholesterolemia } \\
\text { Cardiovascular }\end{array}$ & 0.234 & 0.104 & 0.004 & -0.013 & -0.083 & 0.252 & 0.202 & 0.236 & -0.189 & -0.249 \\
\hline Disease & -0.030 & -0.101 & 0.126 & 0.104 & -0.070 & -0.025 & -0.102 & -0.088 & -0.116 & -0.055 \\
\hline \multicolumn{11}{|c|}{ ^ Significant after Benjamini-Hochberg adjustment } \\
\hline \multicolumn{11}{|c|}{${ }^{* * *} p \leq 0.005$ level (2-tailed). } \\
\hline \multicolumn{11}{|c|}{${ }^{* *} p \leq 0.01$ level (2-tailed). } \\
\hline \multicolumn{11}{|c|}{$p \leq 0.05$ level (2-tailed) } \\
\hline
\end{tabular}


Table 3. (a) Spearman correlation values ( $r$ values) between MRI white matter signal abnormality and cerebrospinal fluid flow variables; and (b) Spearman partial correlations ( $r$ values), controlling for age and hypertension, between MRI white matter signal abnormality and cerebrospinal fluid flow data. $(n=101)$
(a) T2 WM-SAV
$0.201^{*}$

DAWM volume
DAWM+T2 WM-SAV
T2 PVL-SAN
T2 DWM-SAN
0.183
$-0.228^{* \wedge}$
$0.285^{* * \star \wedge} \quad 0.239^{* \wedge}$
$-0.264^{\star \star \wedge}$
0.188
$-0.296^{* * * \wedge}$
$-0.290^{* * * \wedge}$
$0.377^{\star \star \star \wedge} \quad 0.343^{\star \star \star \wedge}$
$-0.311^{* * * \wedge}$
0.187
$-0.195$
$0.373^{\star \star \star \wedge} \quad 0.338^{\star \star \star \wedge}$
$-0.318^{\star \star \star \wedge}$
T2 DWM-SAN
0.131
$-0.290^{\star \star \star \wedge}$
$0.234^{\star \wedge}$
0.164
$-0.160$
(b) T2 WM-SAV
0.065
$-0.199$
0.198
$0.242^{\star \wedge}$
$-0.178$
DAWM volume
$0.260^{*}$
$-0.294^{* \wedge}$
$-0.004$
DAWM+T2 WM-SAV
0.226
$-0.275^{*}$
$0.406^{* * \star \Lambda}$
$0.342^{* * \star}$
$-0.268^{*}$
T2 PVL-SAN
0.233
$-0.157$
$0.377^{\star * * \wedge}$
$0.309^{* \wedge}$
$-0.247^{*}$
T2 DWM-SAN
0.107
$-0.313^{* * \wedge}$
$0.309^{* * \wedge}$
0.160
$-0.131$
$-0.063$

NNF

NPF

PPV

PNV

^ Significant after Benjamini-Hochberg adjustment

${ }^{* * *} p \leq 0.005$ level (2-tailed).

$* * 0.01$ level (2-tailed).

${ }^{*} p \leq 0.05$ level (2-tailed). 
Table 4. Results of multiple linear regression analyses for all subjects $(n=101)$. Dependent variables were T2 WM-SAV, DAWM volume, DAWM volume+T2 WM-SAV, T2 PVL-SAN and T2 DWM-SAN. Independent variables were age, BMI, hypertension, hypercholesterolemia, heart disease, diabetes, smoking, NF, NNF, NPF, PPV and PNV.

\begin{tabular}{|c|c|c|c|c|c|c|c|}
\hline $\begin{array}{l}\text { Dependent } \\
\text { Variables }\end{array}$ & $\begin{array}{l}\text { Independent } \\
\text { Variables }\end{array}$ & $\begin{array}{c}\text { Unstandardized } \\
\text { Coefficients } \\
\text { B }\end{array}$ & $\begin{array}{l}\text { Unstandardized } \\
\text { Coefficients } \\
\text { Std. Error }\end{array}$ & $\begin{array}{l}\text { Standardized } \\
\text { Coefficients } \\
\text { Beta }\end{array}$ & $\begin{array}{l}\text { Significance } \\
\text { p value }\end{array}$ & $\begin{array}{l}\text { Adjusted } \\
\text { r squared }\end{array}$ & $\begin{array}{c}\text { Statistical } \\
\text { power }\end{array}$ \\
\hline \multirow[t]{2}{*}{ T2 WM-SAV } & Constant & -773.503 & 385.881 & & 0.048 & 0.081 & $84.8 \%{ }^{*}$ \\
\hline & Age & 25.171 & 8.032 & 0.300 & 0.002 & & \\
\hline \multirow[t]{3}{*}{ DAWM volume } & Constant & 120.875 & 220.958 & & 0.586 & 0.185 & $99.2 \%^{*}$ \\
\hline & Hypertension & 794.068 & 284.530 & 0.310 & 0.007 & & \\
\hline & NPF & 15.923 & 6.610 & 0.268 & 0.019 & & \\
\hline \multirow[t]{3}{*}{ DAWM+T2 WM-SAV } & Constant & -1395.541 & 620.481 & & 0.027 & 0.160 & $97.9 \%^{*}$ \\
\hline & Age & 36.778 & 10.956 & 0.321 & 0.001 & & \\
\hline & PNV & -124.903 & 60.399 & -0.198 & 0.041 & & \\
\hline T2 PVL-SAN & ns & ns & ns & ns & ns & ns & na \\
\hline Г2 DWM-SAN & ns & $\mathrm{ns}$ & ns & ns & ns & ns & na \\
\hline
\end{tabular}

ns, not significant; na, not applicable

* Statistical power determined using post-hoc analysis 


\section{Figure Captions}

Figure 1. Representation of dirty appearing white matter (DAWM - white arrow) and hyperintense T2 lesions (black arrow) in a 65 yrs old male with hypertension but not altered cerebrospinal fluid (CSF) pulsatility (upper row left) and in 69 yrs old male with altered CSF pulsatility, but no hypertension (upper row right) on fluid attenuated inversion recovery (FLAIR) sequence. The bottom row represent subjects without presence of DAWM or T2 hyperintense lesions in $64 \mathrm{yrs}$ old female (left) and $64 \mathrm{yrs}$ old male (right) who had no hypertension or altered CSF pulsatility.

\section{Figures}
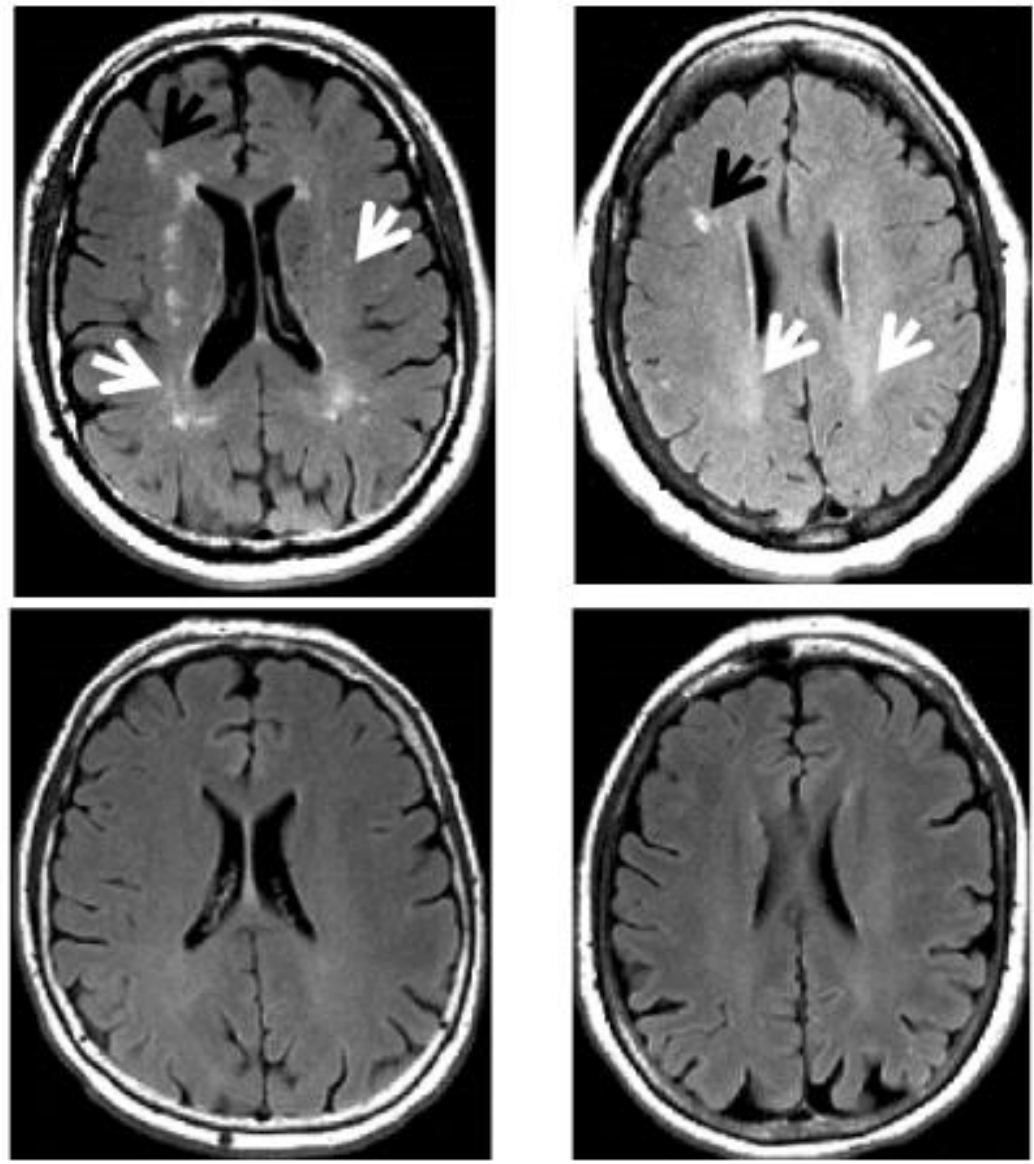

Figure 1. Representation of dirty appearing white matter (DAWM - white arrow) and hyperintense T2 lesions (black arrow) in a 65 yrs old male with hypertension but not altered cerebrospinal fluid (CSF) pulsatility (upper row left) and in 69 yrs old male with altered CSF pulsatility, but no hypertension (upper row right) on fluid attenuated inversion recovery (FLAIR) sequence. The bottom row represent subjects without presence of DAWM or T2 hyperintense lesions in 64 yrs old female (left) and 64 yrs old male (right) who had no hypertension or altered CSF pulsatility. 


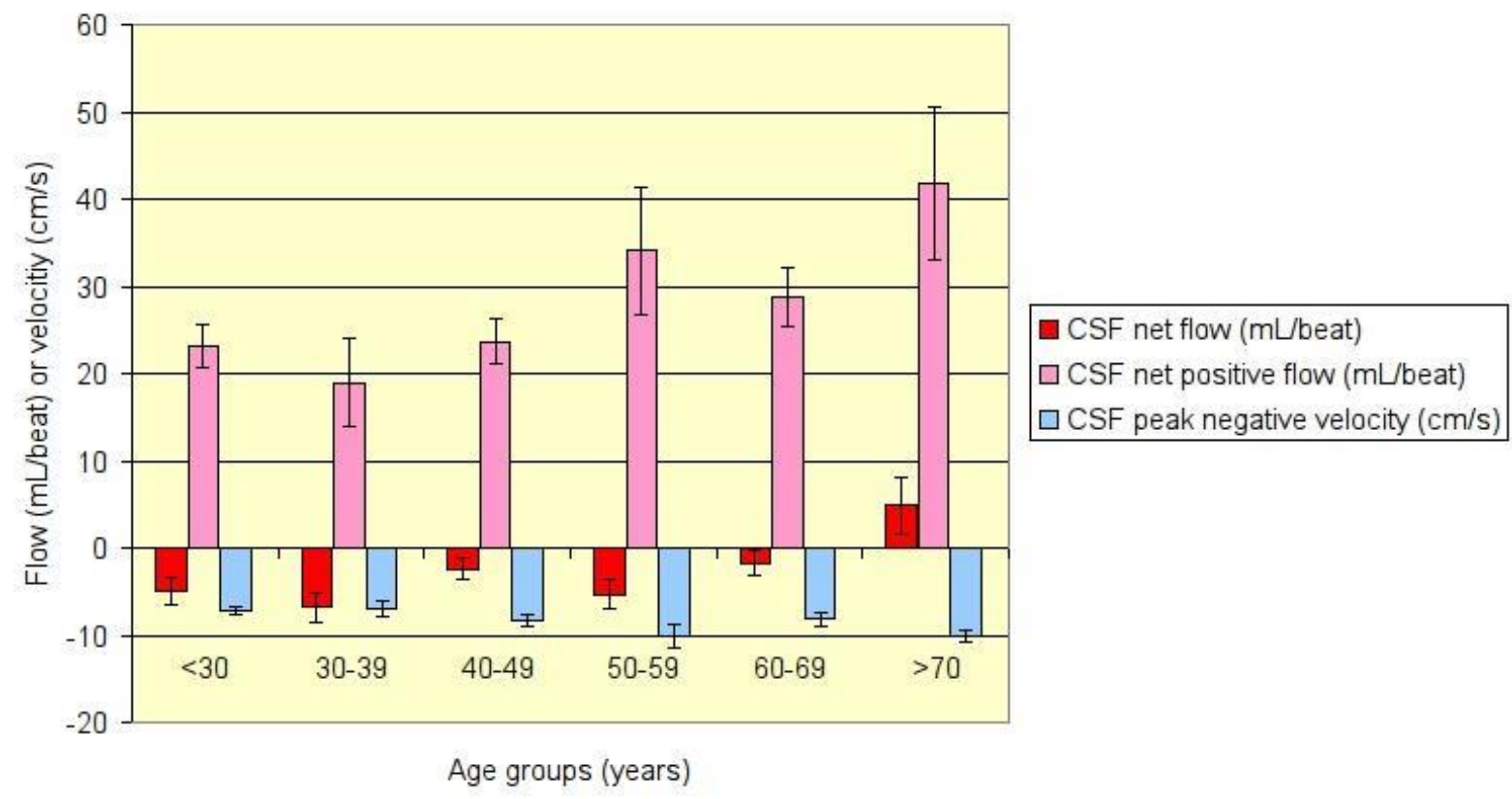

Figure 2. The effect of age on CSF NF, NPF and PNV. Error bars represent one standard error of the mean. 